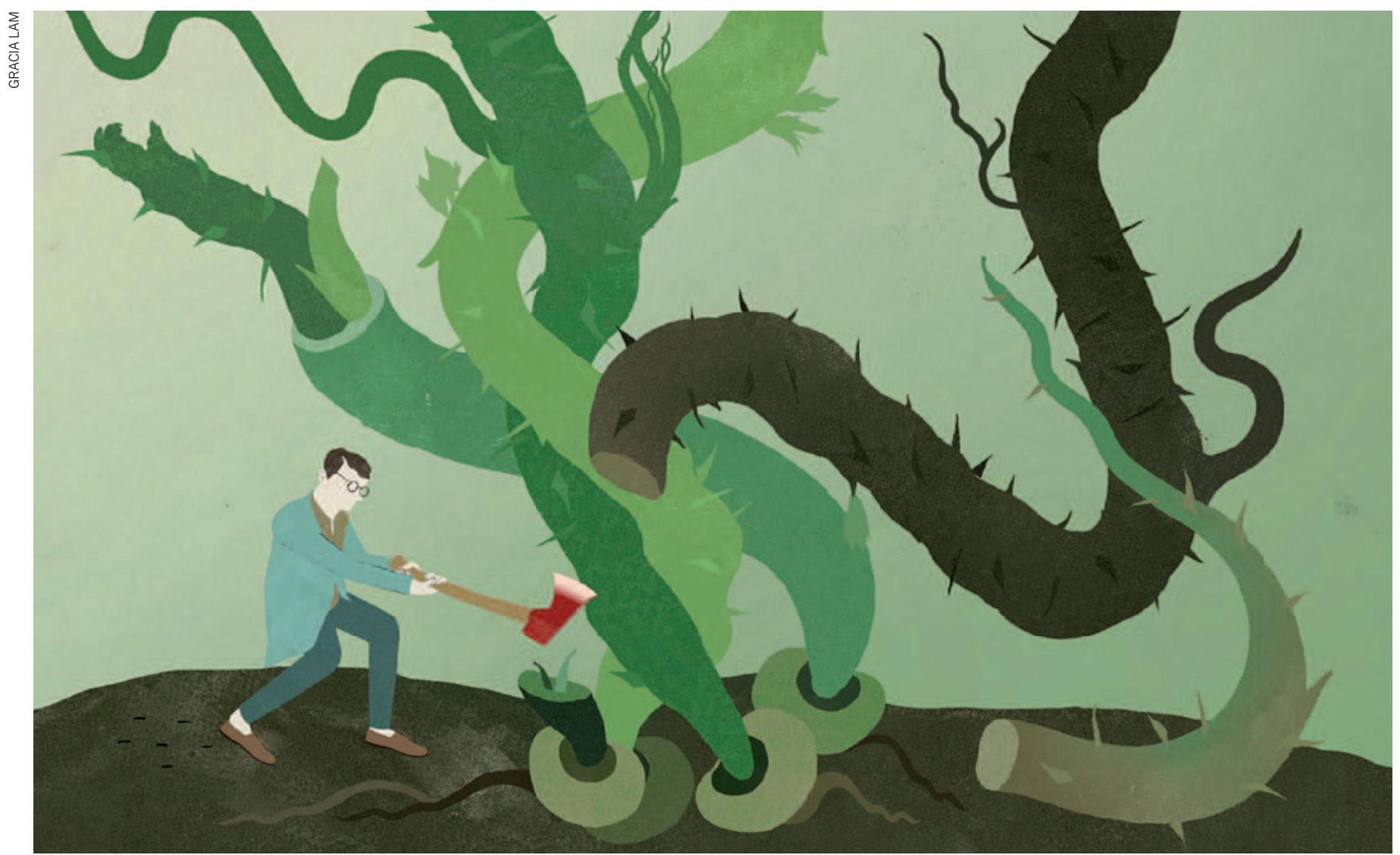

\title{
Testing ground for cancer stem cells
}

\author{
Multiple myeloma is the ideal disease to study a controversial theory about the biology of \\ cancer - and how to cure it.
}

\section{BY CATHRYN DELUDE}

$\mathrm{W}$ hy can drugs send patients with multiple myeloma into seemingly complete remission $80-90 \%$ of the time but never cure them? By all objective measures of the disease - malignant plasma cells in the bone marrow and aberrant M protein in the bloodstream - the cancer is eradicated. Yet patients nearly always relapse.

One possible explanation for this paradox comes from the compelling but controversial cancer stem-cell hypothesis. It states that the drugs kill the malignant plasma cells but leave a reservoir of cancer stem cells that continue growing and can produce more plasma cells. These cancer stem cells are largely untouched by current drugs, says oncologist William Matsui of the Sidney Kimmel Comprehensive
Cancer Center at Johns Hopkins University in Baltimore, Maryland. If we had a way of killing these cells, he says, we could cure multiple myeloma.

The cancer stem-cell hypothesis contradicts the prevailing view that every cancer cell can create more cancer cells or tumours, a process known as tumorigenesis. Instead, according to the hypothesis, cancer consists of a cellular hierarchy. At the bottom, the bulk of tumour cells can cause disease symptoms but cannot maintain cancer growth or propagate new tumours, and they eventually die off. At the top, the long-lived cancer stem cells can renew themselves indefinitely and replenish bulk tumour cells, generate new tumours, and cause metastasis and relapse. Proponents of the cancer stem-cell idea say it explains why, in multiple myeloma and other cancers, drugs that can seem to miraculously and rapidly shrink tumours do not lead to long-term survival ${ }^{1}$.

There is growing evidence that cancer stem cells exist in several cancers, says Max Wicha, a cancer biologist at the University of Michigan. But there is still no evidence that they can be targeted therapeutically, or that doing so would cure the disease or even prevent a relapse and prolong survival. These ideas can be tested, Wicha and others believe, in patients with multiple myeloma who have used existing drugs to achieve clinical remission, with all their cancerous plasma cells eradicated.

But efforts so far to identify multiple myeloma stem cells have been "loaded with controversy, emotion, scepticism and difference of opinion", says physician-researcher Constantine Mitsiades of the Dana-Farber Cancer Institute in Boston, Massachusetts, who is studying 

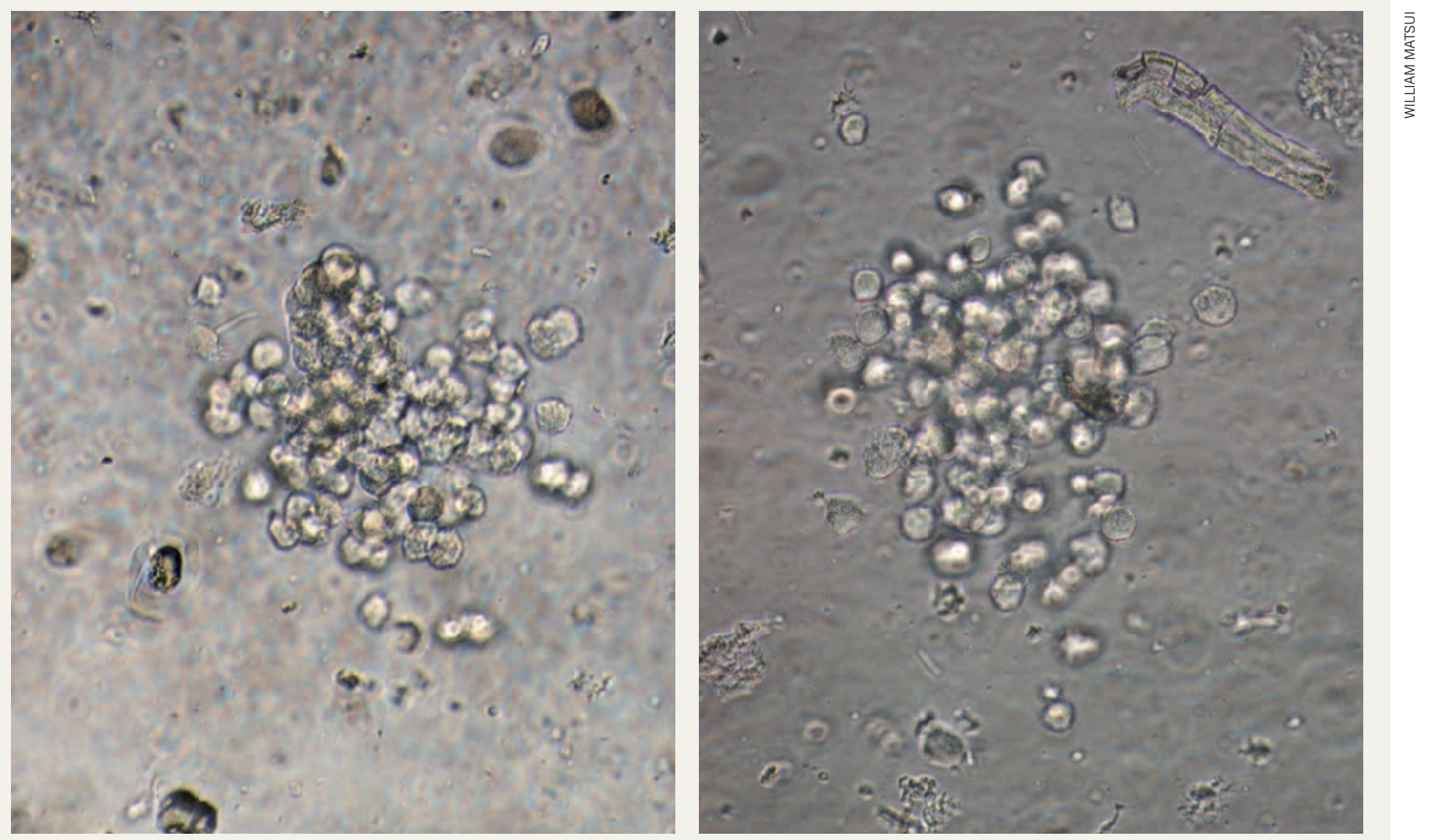

Some researchers believe that myeloma cells like these are derived from cancer stem cells.

these cells in the context of drug resistance in multiple myeloma.

\section{TESTING THE THEORY}

The concept of cancer stem cells was first tested experimentally in 1994, when John Dick, a cancer researcher at the Hospital for Sick Children in Toronto, Canada, transplanted human leukaemia cells into mice with suppressed immune systems that would not reject human cells. Only certain cells could recapitulate the original cancer, with its varied cell types, in a mouse. Harvesting these tumour cells and transplanting them into new mice again and again showed which cells could continue producing tumours without burning out. This method, called serial transplantation, is a functional assay that is still the standard for demonstrating two essential properties of cancer stem cells: the capacities to recapitulate the original tumour and to self-renew.

In the early days, Matsui explains, people thought that cancer stem cells were either normal stem cells that had turned cancerous, or primitive, undifferentiated progenitors that could give rise to many differentiated cell types, and that they must be exceedingly rare in the tumour. These assumptions led to confusion and debates about whether cells that were more differentiated, or less rare, could be considered cancer stem cells - a controversy that is particularly relevant to multiple myeloma. But researchers now generally agree

that many properties attributed to normal stem cells - such as immaturity, quiescence, rareness, embryonic gene expression, rapid proliferation and drug resistance - are not essential to the functional definition of a cancer stem cell. If a cell can recapitulate the disease and has self-renewal properties, it is for all intents and purposes a cancer stem cell ${ }^{1}$.

The central question in multiple myeloma is whether the cells with these properties - the ability to recapitulate the disease and to self-

\section{Proponents} of the cancer stem-cell idea say it explains why drugs that shrink tumours do not lead to long-term survival.

cal implications because it may take different kinds of drug to target plasma cells and B cells ${ }^{2}$.

By the late 1990s, several labs were using variations of the serial transplantation method on multiple myeloma. Some of the first transplant experiments refuted the notion of a subpopulation of myeloma cancer stem cells that is distinct from the plasma cells. Joshua Epstein and Shmuel Yaccoby, cancer cell biologists at the University of Arkansas for Medical Sciences in Little Rock, put human myeloma cells into human fetal bone fragments that had been implanted in immunodeficient mice. They found that human myeloma plasma cells can recapitulate the disease in these mice (see 'Towards a myeloma mouse', page S38).

"All myeloma plasma cells have the potential to proliferate under certain conditions," Epstein says, "and when they do proliferate they change their phenotype to something that other people consider a stem cell." But he does not consider these cells to have self-renewal properties. Instead, he thinks they can maintain the disease and cause relapse because of the capacity of different plasma cells to temporarily acquire the ability to divide. Interestingly, the ability of cells to go in and out of various stem-cell-like states has recently been observed in other cancers.

\section{TURNING TO B CELLS}

Other researchers point to potential problems with the methodology, however. Linda Pilarski, an experimental oncologist at the University of Alberta in Edmonton, Canada, suggests that the fetal bone environment used by Epstein somehow supports the growth of myeloma plasma cells that normally live in adult bones. "The problem is, when you look in the bone marrow, plasma cells do not divide, so the cancer growth must come from an earlier cell that does divide," she says. 
That earlier cell must come from a B-cell progenitor that gives rise to the plasma cells. B cells produce antibodies to a specific antigen, and one type of B cell, the memory B cell, provides long-term or even lifelong immunity to a particular antigen. It does so by continuing to give rise to new plasma cells that keep churning out the antibody to that antigen. Specifically, in response to an antigen, B cells undergo genetic mutations in which they rearrange an immunoglobulin gene in a unique sequence, conferring the 'memory' to memory B cells. The plasma cells derived from these memory $B$ cells inherit the sequence and make antibodies specific to the antigen. In healthy people, the plasma cells produce different antibodies, but in patients with myeloma, all the plasma cells produce the same clone of an antibody: the $\mathrm{M}$ (monoclonal) protein. Pilarski says that this 'clonotypic' relationship between myeloma plasma cells and the precursor memory B cell could reveal the derivation of all the bulk tumour cells from a putative cancer stem cell.

In the early 2000s, Pilarski and her research partner, Andrew Belch, found that it was not the plasma cells but B-cell progenitors (although not necessarily memory B cells) that could self-renew and recapitulate new multiple myeloma tumours. Some previous research had suggested that B cells were involved in multiple myeloma, but no one had tested this idea, and Pilarski recalls that most researchers still assumed that plasma cells were the sole component of multiple myeloma.

The scepticism about her B-cell results forced her to do a more rigorous molecular analysis. In 2008, Pilarski, Belch and Julia Kirshner, then a postdoctoral fellow in Pilarski's lab, showed that a self-renewing population of $\mathrm{B}$ cells can give rise to myeloma plasma cells in their three-dimensional bone-marrow tissue model ${ }^{3}$. "We think these B-cell precursors really were cancer stem cells," she says, "and that this will be a useful therapeutic model."

Pilarski's next aim is to find a way of destroying the tumorigenic B cells. She is also investigating whether myeloma patients with higher levels of these cells relapse earlier. The preliminary evidence suggests that they do. "That tells you these early cells are very important, and very bad for the patient," she says.

In 2004, Matsui extended Pilarski's earlier findings by further defining the clonogenic myeloma cells as memory B cells. Some researchers objected to calling them stem cells because memory $\mathrm{B}$ cells do not give rise to a variety of differentiated cell types, a characteristic typical of other adult stem cells. However, Matsui points out, unlike other differentiated cells in the body, memory B cells have the

$\rightarrow$ NATURE.COM For some of the latest research on multiple myeloma: go.nature.com/za4zy7 and earlier memory B cells, there can be no doubt that whatever the myeloma stem cell is, memory B cells are involved somehow. The uncertainty is whether the cancer stem cells started out as memory B cells and then de-differentiated to be more like the earlier B-cell progenitors, or whether they came from plasma-like cells that acquired some stemcell-like properties but still retain the tell-tale genetic mutations of memory B cells.

\section{SCRATCHING THE SURFACE}

Further debate surrounds the combination of cell surface proteins, or markers, present on the putative myeloma stem cells. Researchers use these markers to sort cells for serial transplantation and other experiments, and to determine which are tumorigenic. Mature plasma cells, for example, have the CD138 marker, whereas CD20 and CD19 are the badges of a B cell. Matsui has shown that the myeloma stem cells lack CD138 but have CD20 and CD19, as well as CD27, which is found on memory B cells. However, these markers do not completely overlap with those identified by other labs.

\section{If a cell can recapitulate the disease and has self-renewal properties, it is for all intents and purposes a cancer stem cell.}

These disparate results may arise partly because different labs use different methods to investigate different stem-cell properties - they may be looking at the same phenomenon but from different angles. Researchers in other cancers have found that cancer cells can shed and acquire stem-cell markers depending on their growth cycle, and on such factors as their proximity to the microenvironment, the activity of the immune system, and exposure to drugs. This may be even more common in myeloma because there are so many distinct stages of the disease. Matsui says it is possible that markers differ not just from person to person, but also within the same person during the course of the disease.

The plasticity of cell surface markers could impede the translation of work to target cancer stem cells into the clinic. "How can clinical trials proceed without reliable markers to indicate if a therapy is targeting cancer stem cells?" asks Wicha. It is likely but not proven, he says, that in curable cancers such as testicular cancer, drugs happen to target both the bulk tumour cells and the cancer stem cells. Furthermore, autologous stem-cell replacement - the only treatment that has ever cured multiple myeloma - probably eliminates cancer stem cells. Despite achieving good results, the stem-cell procedure is generally considered a last resort, because of the risks it entails. "Everybody would greatly prefer if you could use treatments that didn't require a bone-marrow transplant," Wicha says. Many researchers are now investigating whether any drugs, or a combination of drugs, can target myeloma stem cells, or whether they are missing the target and new agents are needed.

\section{A MEASURE OF SUCCESS}

Gauging the success of a cancer stem-cell therapy poses its own problems. A clinical response to a drug is typically measured by tumour shrinkage. But the point of cancer stem-cell therapy is to prevent relapse and metastasis and to prolong survival - all outcomes that could take lengthy, large-scale clinical trials to demonstrate. It is therefore important to have an interim measure, such as reliable biomarkers to identify cancer stem cells. In this respect, multiple myeloma has another advantage: researchers can take repeat biopsies from patients' bone marrow and blood more easily than they can from a solid tumour in a lung, breast or brain. Indeed, it is common to take repeat biopsies to track the progress of the disease, and researchers can use these biopsies to track the effect of therapies on cancer stem cells. "This puts us way ahead of the curve compared to solid tumours," says Matsui, and this is one reason why some of the first trials to test the cancer stem-cell theory are in multiple myeloma.

Matsui discontinued one effort using rituximab, an antibody to CD20 that is used to treat lymphomas, after a small, 21-person phase II trial showed no improvement in progressionfree survival compared with what might be expected with autologous stem-cell transplantation. Follow-up analysis showed that although the antibody targeted the putative myeloma stem cells, it did not kill them effectively. Matsui's collaborators are now analysing whether the therapy reduced the burden of circulating $M$ protein or the number of putative myeloma stem cells in the patients' samples. Matsui and another team are separately using antibodies to CD20 linked to a radioactive molecule to make them more effective. Other monoclonal antibodies targeting other markers are also in development.

Killing myeloma stem cells will not be a replacement for therapies that target malignant plasma cells, which could kill the patient before the cancer stem-cell therapy has time to work. But targeting the cancer stem cells may turn short-term recoveries into long-term, disease-free survival. From a research standpoint, Wicha says, proving the cancer stem-cell theory in multiple myeloma is exciting because it could lead to a validation of the model for other cancers. There is increasing evidence that chasing cancer stem cells will ultimately be fruitful, he says. But the more we learn, the more we realize it is not straightforward.

Cathryn Delude is a science writer based in Andover, Massachusetts.

1. Clarke, M. F. et al. Cancer Res. 66, 9339-9344 (2006).

2. Brennan, S. K. \& Matsui, W. J. Mol. Med. 87 1079-1085 (2009).

3. Kirshner, J. et al. Blood 112, 2935-2945 (2008). 